\title{
Weakened atmospheric energy transport feedback in cold glacial climates
}

\author{
I. Cvijanovic, P. L. Langen, and E. Kaas \\ Centre for Ice and Climate, Niels Bohr Institute, University of Copenhagen, Juliane Maries Vej 30, \\ 2100 Copenhagen, Denmark
}

Received: 10 March 2011 - Published in Clim. Past Discuss.: 13 April 2011

Revised: 12 September 2011 - Accepted: 19 September 2011 - Published: 6 October 2011

\begin{abstract}
The response of atmospheric energy transport during Northern Hemisphere cooling and warming from present day (PD) and Last Glacial Maximum (LGM) conditions is investigated using sea surface temperature anomalies derived from a freshwater hosing experiment. The present day climate shows enhanced sensitivity of the atmospheric mid-latitude energy transport compared to that of the LGM, suggesting its ability to reorganize more easily and thereby dampen high latitude temperature anomalies that may arise from changes in the oceanic transport. This effect is found to be a result of both the atmospheric and surface flux response. The increased PD transport sensitivity relative to that of the LGM is linked to a stronger dry static energy transport response which, in turn, is mainly driven by larger changes in the transient eddy heat flux. In comparison, changes in midlatitude latent heat transport play a minor role in the overall transport sensitivity.
\end{abstract}

\section{Introduction}

Proxy climate data from around the world show enhanced variability, such as the presence of Dansgaard-Oeschger events, during glacial periods compared to the interglacials (Greenland ice cores, NGRIP Members, 2004; Antarctic ice cores, EPICA Members, 2006; Cariaco Basin sediment cores, Peterson et al., 2000; Asian cave stalagmites, Wang et al., 2001; and Arabian Sea sediment cores, Banakar et al., 2010). Changes in the oceanic circulation and halocline stability (due to, e.g. ice discharge or meltwater events) are considered a potential driver of these events as inferred from the ocean sediment data and modeling studies (Keigwin et al., 1994; Broecker, 1994; Dahl et al., 2005). Although the

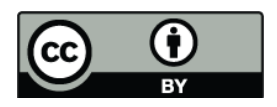

Correspondence to: I. Cvijanovic (ivanacv@nbi.ku.dk) role of the atmosphere is often considered secondary in this regard, various atmospheric and surface feedbacks, such as clouds, albedo, atmospheric energy transport, can enhance or weaken the initial anomaly in the oceanic state. The focus of this study is on a presumably negative feedback, namely the atmospheric heat transport and its response to high northern latitude temperature anomalies under warm interglacial and cold glacial conditions.

Numerous modeling studies have addressed communication between high and low latitudes, showing that changes in ice cover, the Atlantic Meridional Overturning Circulation (AMOC) or generally antisymmetric interhemispheric heating in the high latitudes can induce a displacement of the Intertropical Convergence Zone (ITCZ) (Zhang and Delworth, 2005; Chiang and Bitz, 2005; Broccoli et al., 2006; Chiang et al., 2008). Trenberth and Caron (2001) and Wunsch (2005) have demonstrated a dominance of atmospheric over oceanic transport in the extratropics. Nevertheless, possible changes in the sea ice extent due to oceanic transport variations, and their further influence on the atmospheric circulation make the ocean a relevant trigger for abrupt climate shifts despite the small high latitude ocean heat transport.

The sensitivity of the atmospheric energy transport to global mean temperature and meridional temperature gradients was previously studied by Caballero and Langen (2005) in a series of aquaplanet simulations. They found atmospheric heat transport independent of global mean temperature in cases when the global mean temperature was high and meridional temperature gradient low. These climate states were also the ones in which sensitivity to temperature gradient was the largest. Cheng et al. (2007) focussed on the atmospheric and oceanic heat transport responses due to the AMOC slowdown under LGM and PD conditions. They found the oceanic heat transport largely compensated by the atmospheric heat transport with midlatitude transient eddy transport playing an important role for the overall atmospheric response in both climates. Hwang and

Published by Copernicus Publications on behalf of the European Geosciences Union. 
Frierson (2010) investigated the atmospheric poleward energy transport under global warming and found the change in atmospheric moisture content and latent heat transport to be the main cause for the total atmospheric transport increase with the dry static energy transport playing a smaller, compensating role.

This study investigates the sensitivity of the atmospheric energy transport to meridional temperature gradients under glacial and interglacial climates. In contrast to the previous studies that compared the transport response in the two climates, we address its actual sensitivity and its ability to feed back negatively on the imposed changes. Variations in temperature gradients were imposed by changes in sea surface temperatures (SST) and sea ice lines, reflecting the sea surface response to oceanic heat transport variations. A weakened atmospheric transport sensitivity, i.e. a less negative atmospheric feedback, would imply a climate state with the atmosphere less capable of damping high latitude temperature anomalies arising from changes in the oceanic transport. In this case, the climate is thus likely to be influenced more strongly by excursions in the oceanic circulation. In an atmospheric general circulation model (AGCM), high latitude SST anomalies are applied mainly in the North Atlantic mimicking the effect of changing AMOC strength. The sensitivity of the atmospheric response to different sea ice extents and SSTs is then tested with the anomalies applied in two background climates, PD and LGM.

\section{Experimental configuration}

The atmospheric transport sensitivity is tested using the National Center for Atmospheric Research's CCM3 (Kiehl et al., 1998), employing T42 horizontal resolution with 18 levels in the vertical. This AGCM was run in prescribed SST mode with both PD SSTs (Shea et al., 1992) and LGM SSTs (CLIMAP, 1994) in order to obtain the two control simulations. LGM topography and land mask is taken from the ICE-5G reconstruction of Peltier (2004). Experiments have been run for 30 years and averages over the last 20 years are used in the analysis. The main parameters describing the experiments are given in Table 1.

The performance of the model dynamics was analyzed by Hurrell et al. (1998) and it was found that CCM3 depicts the main storm tracks and transient eddy flux fields reasonably well. Differences occur in the winter over the North Pacific, where the simulated transient eddy heat flux maximum is too strong and displaced to the north. The simulated transient kinetic energy is somewhat underestimated, especially over the summer hemispheres. Magnusdottir and Saravanan (1999) analyzed the meridional heat transport in CCM3. Both in terms of peak value and the latitude of maximum atmospheric transport, their results compare well with those obtained by Trenberth and Caron (2001) based on reanalysis data.
Table 1. Experiment summary, $x$ is the strength factor used to multiply the anomaly $(x=0.5,1,1.5,2)$, ANO - anomaly from ECBiltCLIO experiments.

\begin{tabular}{llll}
\hline experiment & $\begin{array}{l}\mathrm{GHG} \\
\left(\mathrm{CO}_{2}, \mathrm{~N}_{2} \mathrm{O}, \mathrm{CH}_{4}\right)\end{array}$ & $\begin{array}{l}\text { orbital forcing } \\
\text { (obliq., lve, eccen. })\end{array}$ & SST \\
\hline PD_ctrl & $\begin{array}{l}280 \mathrm{ppm}, 270 \mathrm{ppb}, \\
760 \mathrm{ppb}\end{array}$ & $\begin{array}{l}23.446^{\circ}, 102.04^{\circ}, \\
0.016724\end{array}$ & Shea et al. \\
& PD_ctrl & PD_ctrl & PD_ctrl + x · ANO \\
PD_px & & & PD_ctrl $-\mathrm{x} \cdot$ ANO \\
PD_mx & & $22.949^{\circ}, 114.42^{\circ}$, & CLIMAP \\
LGM_ctrl & $200 \mathrm{ppm}, 190 \mathrm{ppb}$, & \\
& $350 \mathrm{ppb}$ & 0.018994 & LGM_ctrl + x · ANO \\
LGM_px & LGM_ctrl & LGM_ctrl & LMG_ctrl $-\mathrm{x} \cdot$ ANO \\
\hline LGM_mx & & &
\end{tabular}

SST anomalies are derived from a freshwater hosing experiment in the intermediate complexity model ECBilt-CLIO (Goosse and Fichefet, 1999; Opsteegh et al., 1998). The ECBilt-CLIO was spun up for 1500 years using present day orbital forcing and pre-industrial GHG levels and this state was used as a starting point for the freshwater hosing experiments. In a manner similar to that of Renssen et al. (2002), a freshwater forcing of $1.5 \mathrm{~Sv}$ was applied to the ECBilt-CLIO for 20 years in the North Atlantic and SST anomalies are calculated from the difference between the resulting on-state after recovery and the off-state of the AMOC (details are given by Wang, 2009). These annually varying SST anomaly fields are multiplied by a series of different strength factors and added to the two background control fields, representing the present day and Last Glacial Maximum, in the CCM3 (Fig. 1a). A total of 18 experiments were performed: two control runs (PD and LGM) and 8 sets of perturbations starting from each. Experiments are named after the strength factor used to multiply the anomaly: prefix $p$ refers to positive anomalies (NH warming) and $m$ to negative anomalies (NH cooling), with strengths ranging from -2 to 2 in steps of 0.5 . The same SST anomalies are applied in both sets of experiments. We limit this study, by construction, to the mechanism of the fast atmospheric response under the two climates and do not consider potential differences in the slower oceanic response between PD and LGM.

In the simulations, a sea surface temperature of $-1.8^{\circ} \mathrm{C}$ (freezing point) is used as the criterion to distinguish between the open ocean points and sea ice, with a fixed thickness of $2 \mathrm{~m}$. Therefore, when preparing the perturbation input fields, surface temperature $\left(T_{\mathrm{S}}\right)$ fields from the control experiments were employed at the ocean points to allow for temperatures below $-1.8^{\circ} \mathrm{C}$ in the presence of sea ice. During summer, however, sea ice $T_{\mathrm{S}}$ may attain values between $-1.8^{\circ} \mathrm{C}$ and $0^{\circ} \mathrm{C}$, which would imply that a sea ice point is mistaken for an ocean point. To avoid this, we subtracted $1.8^{\circ} \mathrm{C}$ from the sea ice points with temperatures above the freezing point of sea water. The SST anomaly fields were then added to these control $T_{\mathrm{s}}$ fields and Fig. $1 \mathrm{~b}$ and $\mathrm{c}$ demonstrates the resulting 

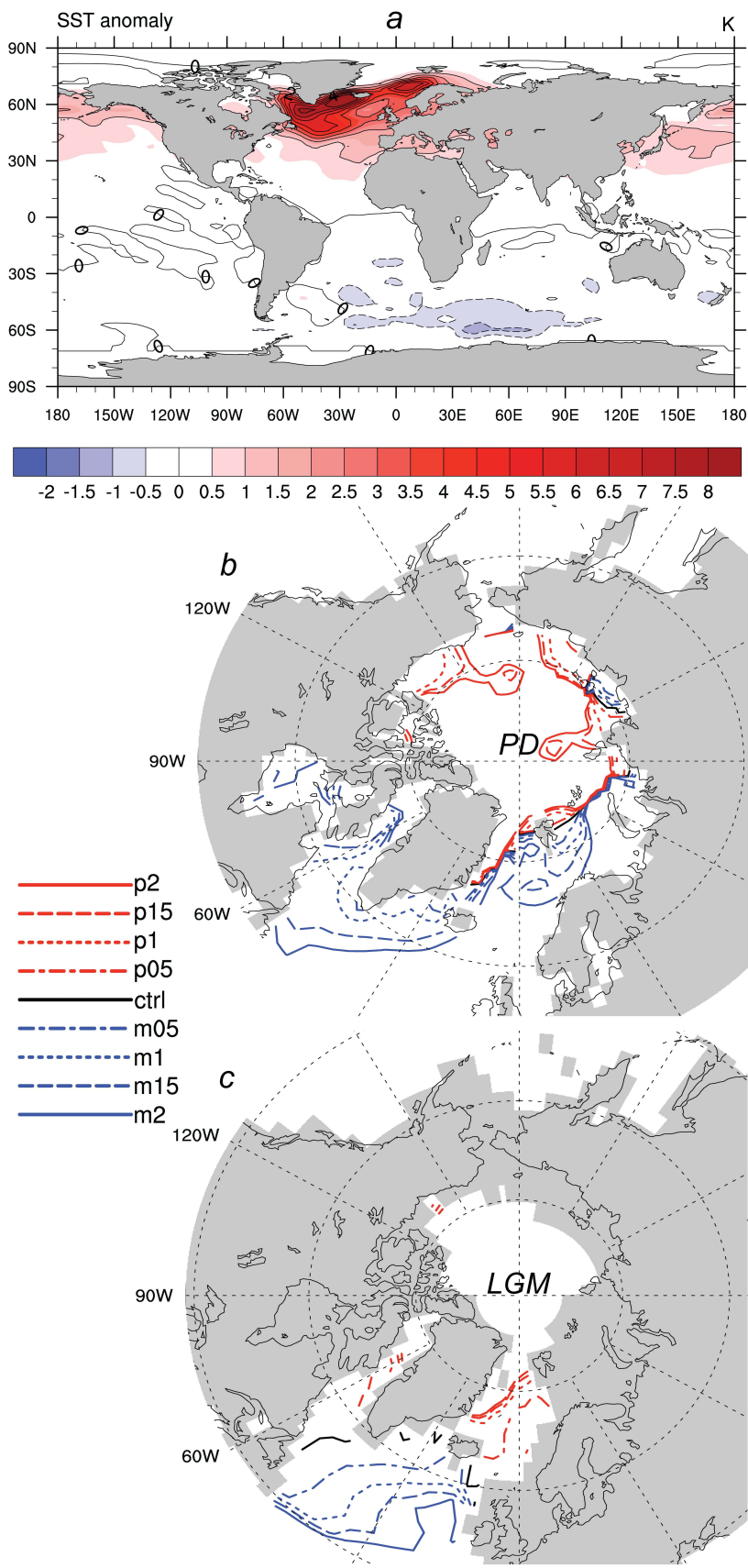

Fig. 1. (a) Annual sea surface temperature anomalies from ECBiltCLIO experiments. Negative anomalies: dashed ( $0.5 \mathrm{~K}$ intervals), positive anomalies: solid ( $1 \mathrm{~K}$ intervals). Panels (b) and (c): $\mathrm{NH}$ summer (JJA) sea ice lines $\left(-1.8^{\circ} \mathrm{C} \mathrm{SST}\right.$ isotherms) for PD and LGM simulations, respectively.

sea ice lines. Note that summer (JJA) is shown here as it is the season with the highest sea ice decline due to the anomalies imposed.

Although we do impose perturbations that tend to realistically represent the effects of changes in the oceanic heat transport, the important point, however, is not the exact SST and sea ice line geometries, which will depend on model and background climate, but the general alteration of the large scale Northern Hemisphere meridional temperature difference (NHTD) obtained in this way. Here we define NHTD as the difference of the average surface temperatures over the areas $0-30^{\circ} \mathrm{N}$ and $30-90^{\circ} \mathrm{N}$, including both land and ocean points. Using the NHTD as a metric enables a comparison between the two climates that significantly differ from each other in their surface conditions.

We consider the NHTD and not the alteration of the implied ocean heat transport in the following sensitivity analysis. This is due to the fact that the SSTs are prescribed in the simulations and there is no possibility of ocean transport feedback. Thus, given a surface temperature perturbation, which in this case is measured by the meridional temperature difference, we investigate the resulting atmospheric heat transport response. The normalization is performed using the surface temperatures as the primary cause of change and not the surface fluxes or, equivalently, the implied ocean heat transport. Of course, the surface fluxes influence the atmospheric state and the atmospheric heat transport, and are in turn influenced by the latter two. Hence, it is important to evaluate how the SST change is communicated to the atmosphere by the surface fluxes and so we complete our analysis by also considering the surface budget imbalance per NHTD.

The SST anomalies applied in the PD and LGM simulations are identical, but the resulting NHTD anomalies are not, because the large LGM sea ice extent with low surface temperatures requires much larger anomalies for open ocean points to emerge. Therefore, the resulting range of the imposed NHTD changes is around $3 \mathrm{~K}$ for LGM simulations and $5 \mathrm{~K}$ for PD. In order to obtain a LGM NHTD anomaly with a value similar to that in the maximum PD warming case (PD_p2) we have included in our analysis an additional experiment in which three times the initial anomaly was added to the LGM background climate (LM_p3). NHTD values for each experiment are summarized in Table 2. Due to the strength of the coupling between surface and surface air temperature, all conclusions in the following remain unchanged if the surface air temperature or lower tropospheric temperature was used instead of surface temperature in the definition of NHTD.

\section{Results}

\subsection{Atmospheric transport sensitivities}

Total northward atmospheric and latent energy transports are calculated from the atmospheric energy and fresh water budgets, respectively. Such implied transport calculations assume a steady state with constant (long term) energy and moisture content in the column, which is the case for the multi-year averages used in this study. In these calculations, monthly mean surface and top-of-atmosphere (TOA) fluxes 
Table 2. Resulting Northern Hemisphere meridional temperature difference (NHTD) from the various experiments.

\begin{tabular}{ll}
\hline experiment & NHTD [K] \\
\hline PD_m2 & 22.6 \\
PD_m1.5 & 22.1 \\
PD_m1 & 21.4 \\
PD_m0.5 & 20.06 \\
\hline PD_ctrl & 20.1 \\
\hline PD_p0.5 & 19.6 \\
PD_p1 & 18.9 \\
PD_p1.5 & 18.4 \\
PD_p2 & 17.8 \\
\hline LGM_m2 & 28.85 \\
LGM_m1.5 & 28.5 \\
LGM_m1 & 28.13 \\
LGM_m0.5 & 27.85 \\
\hline LGM_ctrl & 27.5 \\
\hline LGM_p0.5 & 27.1 \\
LGM_p1 & 26.7 \\
LGM_p1.5 & 26.5 \\
LGM_p2 & 26.1 \\
\hline
\end{tabular}

and precipitation values are used. Whilst the total atmospheric transport is obtained by integrating the atmospheric net energy budget over the latitudes (from the south pole to the given latitude), latent energy transport is calculated considering the fresh water budget obtained as surface evaporation minus precipitation. Northward dry static energy transport (DSE) is obtained as the residual between the total atmospheric and latent heat transports. We do not account for the very small kinetic energy transport and it remains a part of the DSE transport. These calculations have been verified by direct calculation using the individual time step values of vertical integrals of meridional advection of temperature, water vapor and geopotential ( $v T, v q$ and $v \Phi)$, but as these fields were not obtained for all experiments we use the approach described above.

Total transport anomalies at the equator and $30^{\circ} \mathrm{N}$ are plotted as a function of NHTD anomalies in Fig. 2. Imposed NH warming (negative NHTD anomalies) causes a decreased total atmospheric transport, while NH cooling causes an increased transport. Panels a, b and c show total, dry static energy and latent cross-equatorial heat transport for PD (solid circles) and LGM (open circles) warming and cooling simulations. Panels d, e and $\mathrm{f}$ show the same for the transports across $30^{\circ} \mathrm{N}$.

Linear regression coefficients $r c$ (and the corresponding $r^{2}$ values) of transport versus NHTD anomalies provide a measure of the sensitivity of the atmospheric transport. Statistical significance of the results was confirmed by Monte Carlo sampling, giving frequency distributions of regression coefficients differences between the two climates based on yearly samples. The LGM simulations indicate an enhanced total transport sensitivity at the equator and decreased sensitivity at $30^{\circ} \mathrm{N}$ relative to PD (Fig. $2 \mathrm{a}$ and d). The PD simulations show weaker response at the equator with sensitivity increasing northward and peaking in the mid-latitudes, as it can be seen from Fig. 3a and d showing the meridional transport anomalies.

Separation of the total transport into DSE (Fig. 2b) and latent heat (Fig. 2c) components reveals the canceling effect of the two at the equator with DSE giving the sign of the total response in both climates. Slopes for DSE are $0.45 \mathrm{PW} \mathrm{K}^{-1}$ and $0.68 \mathrm{PW} \mathrm{K}^{-1}$ compared to $-0.36 \mathrm{PW} \mathrm{K}^{-1}$ and $-0.47 \mathrm{PW} \mathrm{K}^{-1}$ for latent transport, for the PD and LGM cases, respectively. Both components show stronger cross equatorial response in the LGM climate compared to the PD.

At $30^{\circ} \mathrm{N}$, the PD total response is double that of the LGM with the DSE component being the main cause of the difference (Fig. 2d and e). Compared to the LGM, the PD DSE response is almost three times larger with slopes $0.16 \mathrm{PW} \mathrm{K}^{-1}$ and $0.05 \mathrm{PW} \mathrm{K}^{-1}$ for PD and LGM. Latent energy transport (Fig. 2f) shows lower sensitivity compared to DSE (Fig. 2e) in PD simulations. In contrast to this, in LGM simulations DSE and latent heat transport sensitivities are approximately the same.

An additional experiment, in which three times the initial anomaly was added to the LGM background climate (LM_p3), was performed in order to obtain a NHTD anomaly with a value similar to that in the maximum NH PD warming case (PD_p2). This additional experiment also allows for a comparison between the two climates under similar sea ice extents. This is illustrated in Fig. 2 (in red open circles). The overall slope of the midlatitude LGM response is still much smaller than the PD even when including such an extreme perturbation.

We also tested whether the above findings are valid for the monthly transport anomalies due to the seasonal changes in NHTD (not shown). In comparison with our original experiments with high- to mid-latitude perturbations, the NHTD has much larger variations throughout the course of the year with amplitude close to $30 \mathrm{~K}$. The weakened LGM midlatitude transport sensitivity to NHTD perturbations remains valid for the monthly values: the response is higher in the PD than in the LGM simulation and this becomes even more pronounced in the higher mid-latitudes. DSE transport remains the main contributor to the total transport sensitivity with enhanced influence over latent heat transport.

\subsection{Meridional structure}

To further investigate the atmospheric transport response to the imposed perturbations, we consider the meridional structure of the anomalies (Fig. 3). PD total transport anomalies (Fig. 3a) peak around $35^{\circ} \mathrm{N}$ with larger amplitude than the 

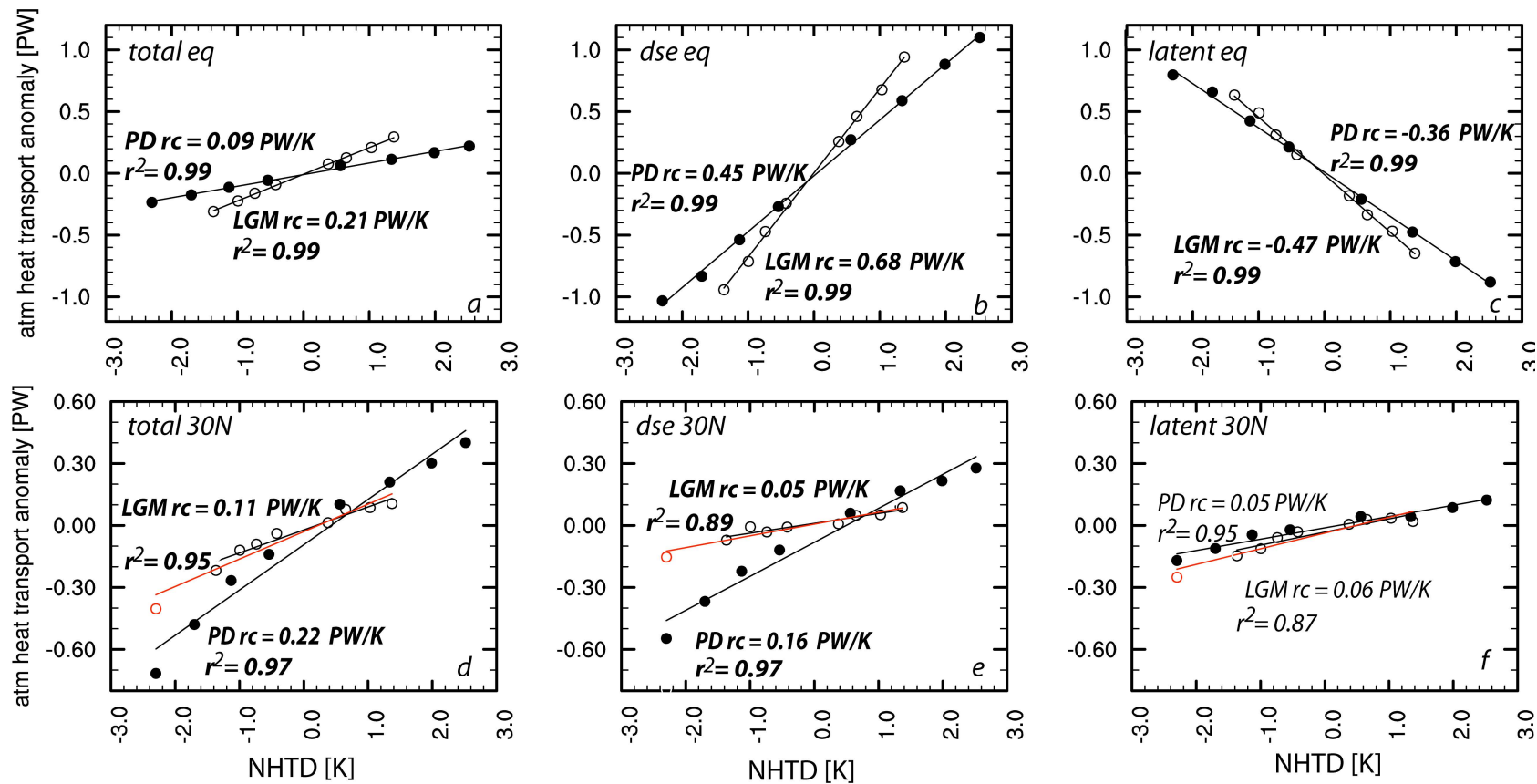

Fig. 2. Atmospheric transport anomalies (PW) at the equator (a, b, c) and $30^{\circ} \mathrm{N}$ (d, e, f) for PD (full circles) and LGM (open circles) simulations as a function of NHTD anomaly (K). Positive/negative NHTD anomalies refer to NH cooling/warming. Linear regression coefficients and $r^{2}$ values of the fit are indicated. Bold font indicates that the difference between PD and LGM slopes is statistically significant at the $95 \%$ level. Red open circle represents the LGM_p3 experiment.
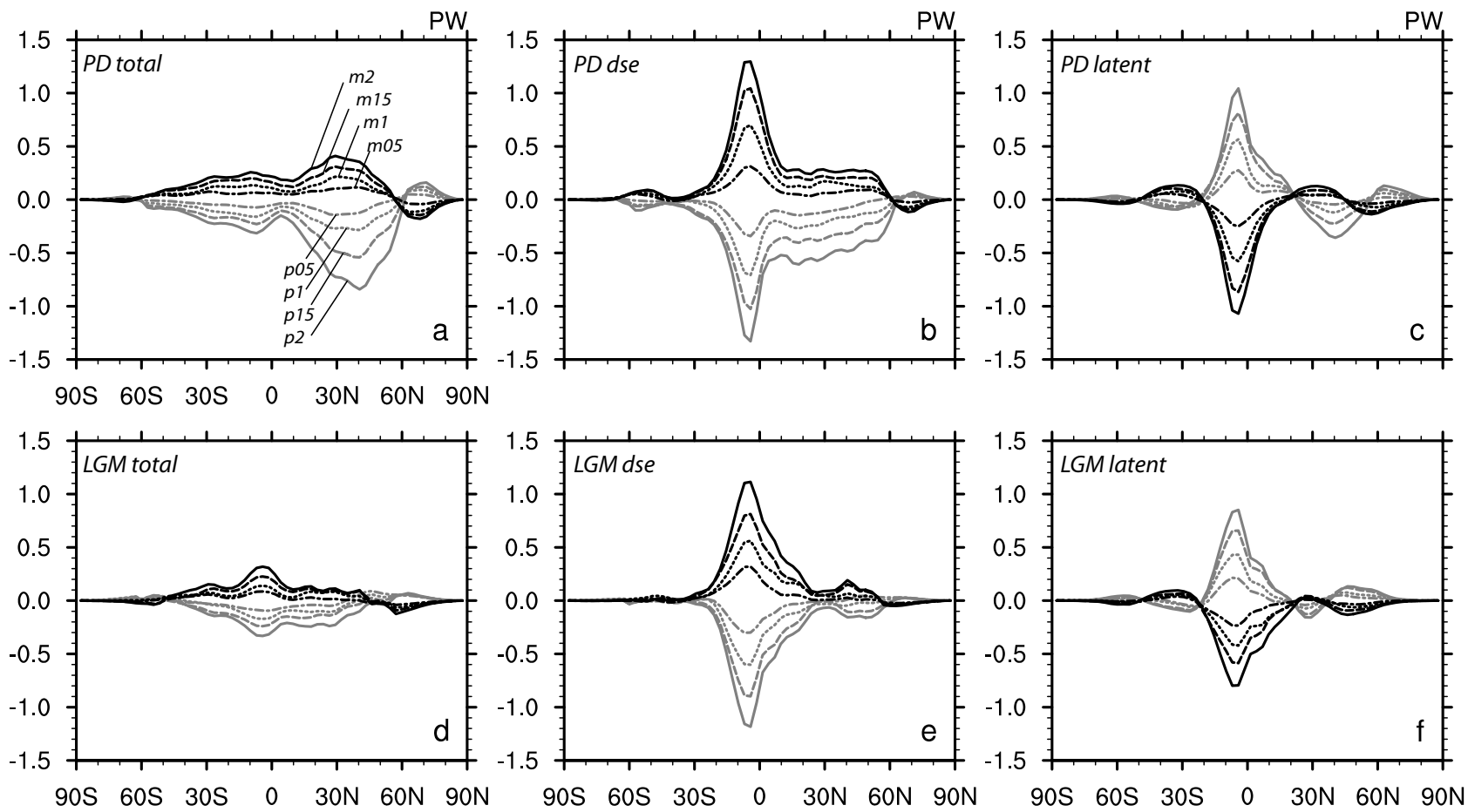

Fig. 3. Atmospheric transport anomalies: total - (a) and (d), DSE - (b) and (e), latent heat - (c) and (f) - for PD (upper plots) and LGM (lower plots) simulations. Black curves: NH cooling simulations $(m 2, m 1.5, m 1$ and $m 0.5)$, grey curves: NH warming simulations ( $p 2, p 1.5$, p1 and $p 0.5)$. 
LGM anomalies (Fig. 3d) whose peak is located close to the equator. Separating the total transport into its two components, we find that the larger anomalies are seen in DSE, both in PD and LGM climates. An interesting feature is the tail in the PD DSE transport anomaly (Fig. 3b) that reaches up to $60^{\circ} \mathrm{N}$. Here DSE and latent heat transport anomalies have the same sign and this is thus the main cause of the extratropical peak in the PD net transport anomaly (Fig. 3a). Northward of $60^{\circ} \mathrm{N}$, we see a negative DSE anomaly as a consequence of decreased temperature gradient between $60^{\circ} \mathrm{N}$ and the area poleward of it. Such features are not present in the LGM and the peak in total atmospheric LGM transport (Fig. 3d) comes from a weaker cancellation between equatorial DSE and latent heat transport changes.

In order to evaluate how the alterations to the atmospheric heat transport (AHT) compare to model-observation biases, we have plotted in Fig. 4 total atmospheric heat transport from our PD control run, together with NCEP and ECMWF (ERA15) data (based on years 1985-1989). Reanalysis-based transport data are derived by Trenberth and Caron (2001). Atmospheric heat transport from the PD_ctrl run is quite close to the atmospheric heat transports from the re-analyses. The anomalies to the re-analysis plots have the same order of magnitude as the anomalies in our perturbed experiments but the locations where they appear to be the strongest are far from the locations of the maximum anomalies in the perturbed experiments. Our perturbations to AHT are small, and in this aspect, they do not force the model to unphysical conditions. The fact that the transport anomalies are much smaller than the model climatologic transports confirms that a simple linear perturbation study is a suitable approach.

\subsubsection{Mid-latitude response}

By means of direct transport calculations, using vertical integrals of meridional advection of temperature and geopotential, dry static energy transport anomalies have been decomposed into their sensible and potential components (not shown). This revealed the canceling effect that the two have, with the sensible heat transport anomaly yielding the sign of the mid-latitude PD DSE response. In contrast, in the LGM simulations both components have much smaller anomalies with the sensible heat transport determining the overall DSE anomaly in lower mid-latitudes and potential energy transport in the higher mid-latitudes. Given that the atmospheric heat transport in mid-latitudes is mainly done by eddies, the different responses of the sensible heat transport in the two climates can be explained by considering the stationary and transient eddy heat flux components. Figures 5 and 6 show stationary $\left[\overline{v^{*}} \overline{T^{*}}\right]$ (upper panels) and transient eddy $\left[\overline{v^{\prime} T^{\prime}}\right]$ (lower panels) meridional heat flux anomalies for PD and LGM simulations, respectively. Square brackets denote zonal means and overbars time means, primes and stars denote deviations from the time and zonal mean, respectively.

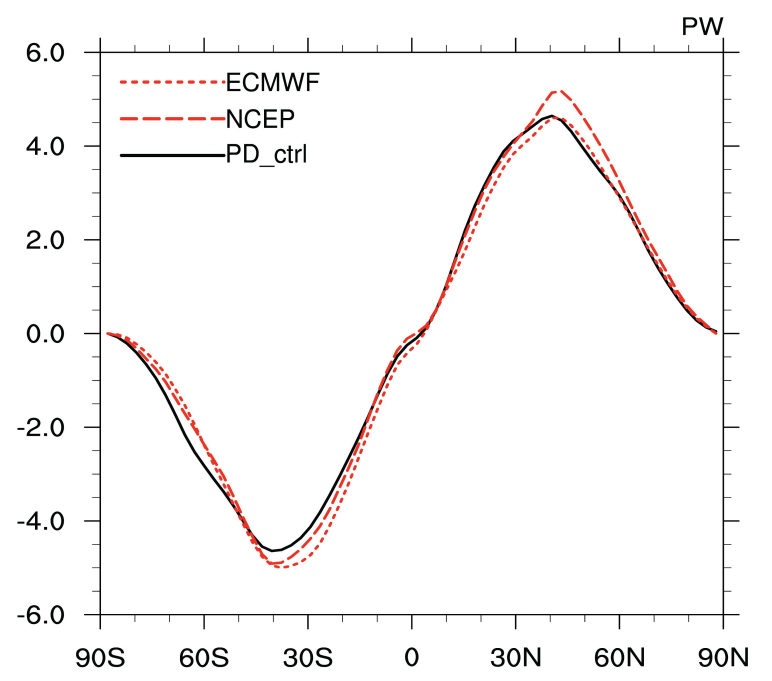

Fig. 4. Atmospheric heat transport for PD_ctrl, ECMWF ERA15 and NCEP data (reanalysis data: February 1985-Apil 1989 annual mean, from Trenberth and Caron, 2001 and Trenberth et al., 2000)

The eddy momentum diagnostics presented here are obtained from the monthly model output, using the decomposition of the total flux into a mean meridional circulation term and stationary and transient eddy flux terms (Peixoto and Oort, 1992). In this manner, total flux and mean meridional circulation terms are obtained directly from the model output, the stationary eddy flux is calculated by subtracting the zonal means while the transient eddy flux is given as the difference between the total flux and the other two terms. We have verified this approach by calculating the transient eddy flux from the daily data (by subtracting the 8 day means from the daily values, but using a smaller sample of years) and arrived at the same conclusions.

We find the glacial control climate to have enhanced stationary eddy heat fluxes compared to the PD control and weaker transient eddy heat fluxes (not shown). A similar result was found by Murakami et al. (2008), who saw increased mid-latitude dry static energy transport during LGM due to increased stationary wave activity. Increased stationary wave activity in LGM has been shown to be caused by the LGM orography (Cook and Held, 1988). Despite the increased stationary eddy heat flux in the glacial control, it is the transient eddy heat flux anomalies that determine the overall eddy heat flux response to the added surface perturbations in both climates (Figs. 5 and 6). With NH cooling, the transient eddy heat flux increases with the equator to pole temperature gradient and conversely, it decreases with $\mathrm{NH}$ warming, but in the LGM simulations this response is weaker than in PD and displaced towards the lower mid-latitudes. Figure 7 , showing the vertically integrated transient and stationary eddy heat transport anomalies, confirms this picture of significantly larger transient transport anomalies (left panel) compared to the stationary transport anomalies (right panel) 

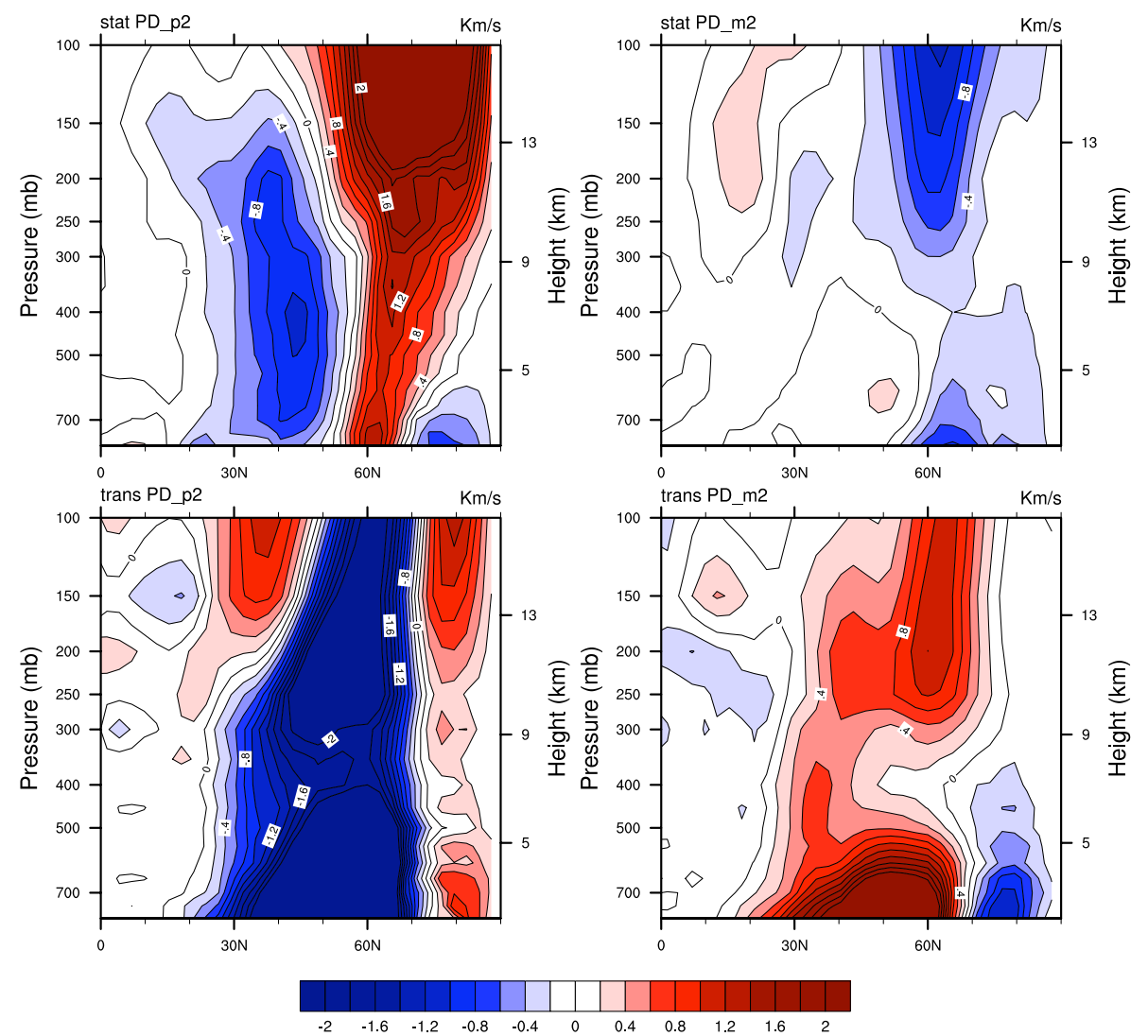

Fig. 5. Stationary (upper panels) and transient eddy (lower panels) annual meridional heat flux anomalies for PD_m2 and PD_p2 simulations.

and the enhanced PD response (black lines) compared to the LGM (red lines).

In conclusion, the cause of the decreased mid-latitude dry static energy transport sensitivity in cold glacial climates is the decreased sensible heat transport sensitivity, which, in turn, is a consequence of the weakened transient eddy heat flux response. In our LGM simulations, we see an enhanced and narrower polar jet (Fig. 8c) and decreased eddy kinetic energy (Fig. 8d) in the North Atlantic compared to the PD simulations (Fig. 8a and b).

Other studies (Li and Battisti, 2008; Donohoe and Battisti, 2009) have noted reduced storminess under glacial conditions despite the stronger Atlantic jet, which is in line with our findings. The dominance of mid-latitude DSE response in AMOC slowdown experiments was also found by Cheng et al. (2007), who also attributed it to increased transient eddy activity. It is important to note the difference to the global warming experiments of Hwang and Frierson (2010), in which latent heat transport dominates the overall response.

Caution should be taken, given that CCM3 overestimates the winter maximum in transient eddy heat flux over the North Pacific (Hurrell et al., 1998). Nevertheless, our analysis focuses on relative changes (compared to the base state) and is in line with existing studies (Cheng et al., 2007; Li and Battisti, 2008; Donohoe and Battisti, 2009) suggesting that the found response is robust.

\subsubsection{Low-latitude effects}

Changes in cross-equatorial energy transports are related to changes in the Hadley cell dynamics and ITCZ shifts. A more northward ITCZ location results in more energy transported to the Southern Hemisphere and vice versa. Dry static energy transport increases towards the cooled hemisphere and latent heat transport towards the warmed one. The location of the crossover between the annual latent heat and DSE transports was found by Trenberth and Stepaniak (2003) to coincide with the time mean location of the ITCZ, and we will exploit that here and use it as a proxy. This is shown in Fig. 9 for the PD and LGM simulations as the intersection of lines with the same dash pattern and color. In PD simulations, the crossover is located in the Northern Hemisphere for all of the perturbations. The position is at $5^{\circ} \mathrm{N}$ in the control, and with increasing perturbation strength it gets displaced northward $\left(6^{\circ} \mathrm{N}\right.$ in PD_p2) or southward $\left(3^{\circ} \mathrm{N}\right.$ in PD_m2), while staying in the NH. In contrast, the LGM time mean ITCZ location is closer to the equator and more sensitive to the imposed perturbations such that it shifts all the 

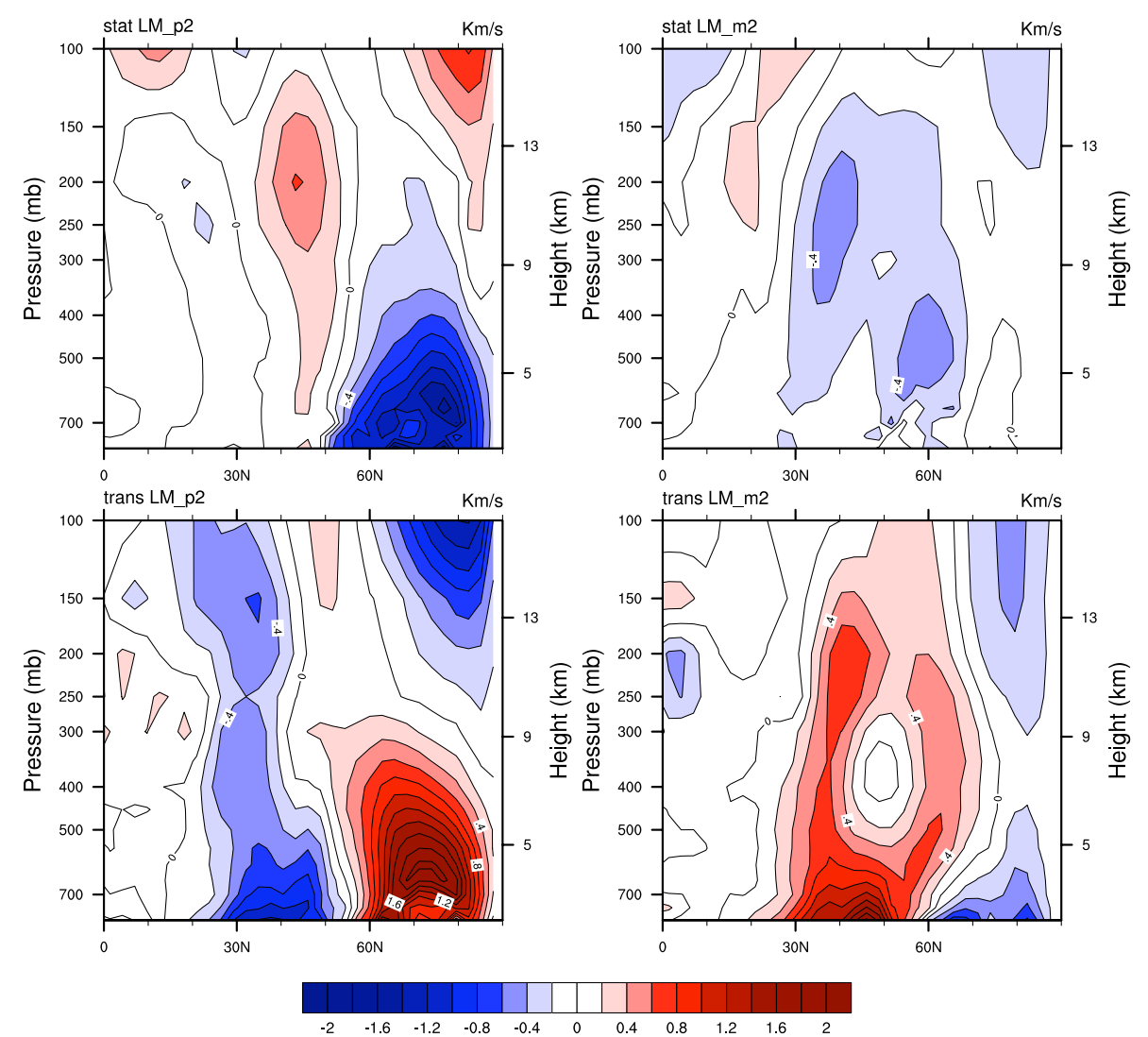

Fig. 6. Stationary (upper panels) and transient eddy (lower panels) annual heat flux anomalies for LGM_m2 and LGM_p2 simulations.
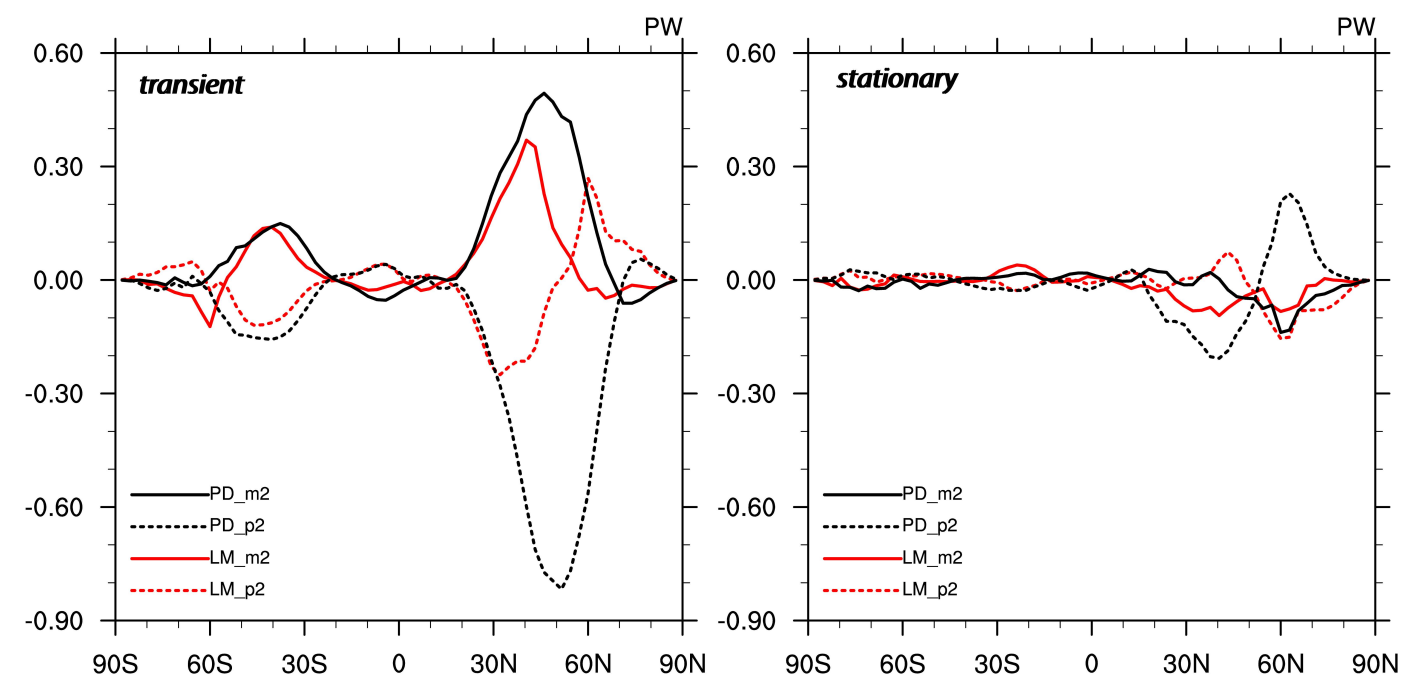

Fig. 7. Vertically integrated transient (left panel) and stationary (right panel) annual eddy heat transport anomalies for PD (black) and LGM (red) simulations.

way to $6^{\circ} \mathrm{S}$ in the cooling simulation (LM_m2) and to $6^{\circ} \mathrm{N}$ in the warming simulation (LM_p2). In comparison to the Trenberth and Stepaniak (2003), (Fig. 1, top panel), we see that the DSE and latent heat transport crossover is located at approximately $5^{\circ} \mathrm{N}$, which fits very well with the location of the crossover in our PD control simulation. 

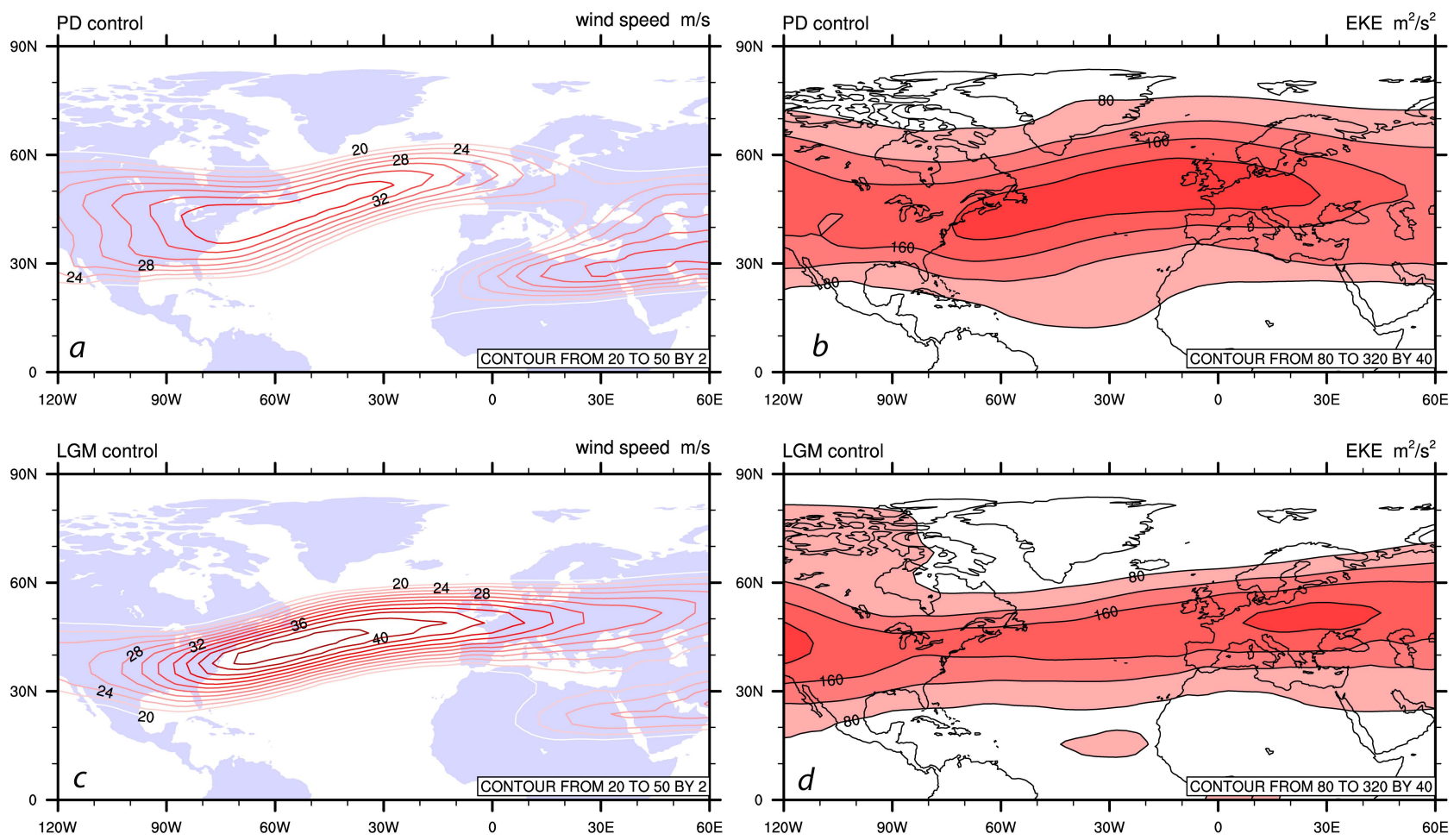

Fig. 8. Annual $200 \mathrm{hPa}$ wind speed - (a) and (c) - and eddy kinetic energy - (b) and (d) - for PD and LGM control climates.
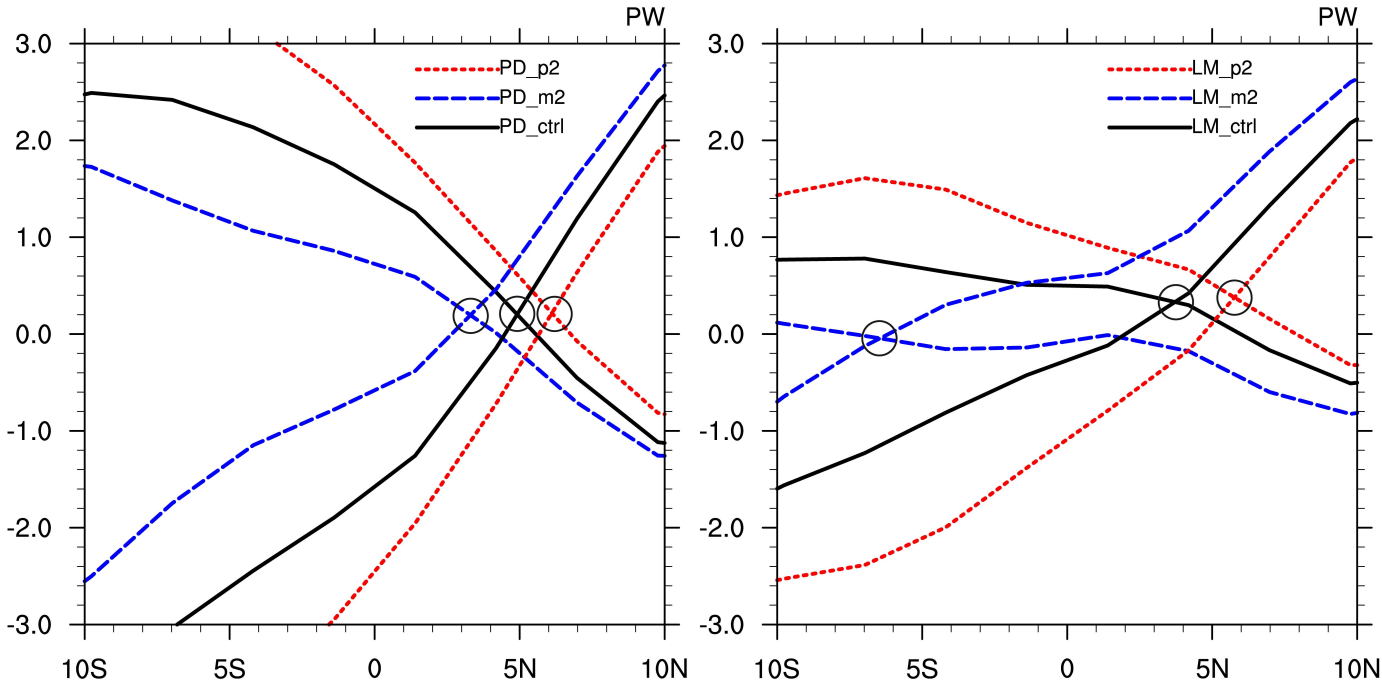

Fig. 9. Locations of annual DSE and latent heat transport crossovers in PD (left panel) and LGM (right panel) simulations.

The fact that the crossover between the two transports colocates with the present day ITCZ does not necessarily carry over to LGM conditions; therefore, this may not be a suitable mean ITCZ location proxy in cold glacial climates. Nevertheless, consideration of the crossovers reveals that the energy transport partitioning under glacial and interglacial conditions is essentially different.
The Hadley cell's efficiency in transporting energy poleward can be inferred from the total gross moist static stability, by considering the ratio between the total energy and mass transports as done in the study by Kang et al. (2009). In our experiments, the Hadley cells are not necessarily located over the same areas in the different simulations. Similarly to the described ITCZ shifts, the meeting points of the northern and southern Hadley cells move more in LGM than 

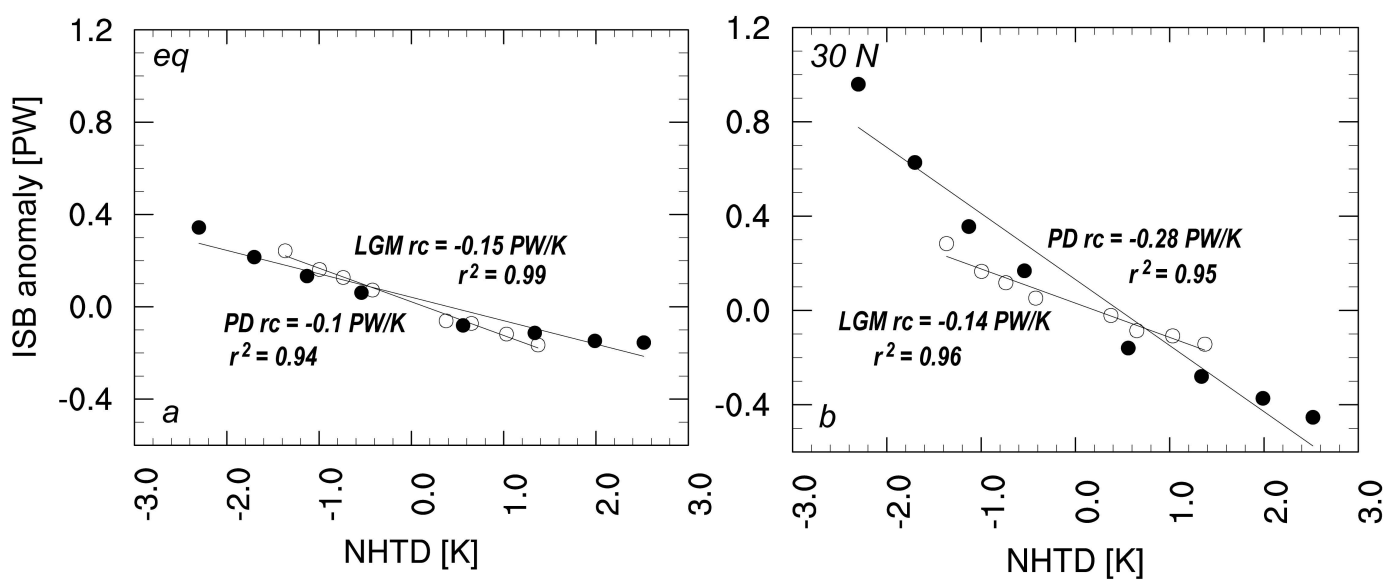

Fig. 10. Integrated surface budget anomalies (ISB), in PW, at the equator (a) and $30^{\circ} \mathrm{N}$ (b) for PD (full circles) and LGM (open circles) simulations as a function of NHTD anomaly (K). As in the Fig. 2, positive NHTD anomalies indicate NH cooling and vice versa. Linear regression coefficients and $r^{2}$ values of the fit are indicated.

in PD simulations, and locations of the Hadley cell maxima can be at different heights or latitudes in different simulations. Such is the case for the annual PD and LGM northern Hadley cells maxima that are located close to $15^{\circ} \mathrm{N}$ but at $700 \mathrm{hPa}$ and $500 \mathrm{hPa}$, respectively. Therefore, instead of specifying a certain latitude and height, we simply consider the point of the maximum of the mass stream function. We then calculate the ratio of the northward energy transport at this latitude and the stream function maximum itself. In this manner, we obtain a measure of the northern Hadley cell efficiency in transporting energy to the mid-latitudes, which is directly comparable for PD and LGM conditions. Such analysis shows that the drier low-level LGM atmosphere increases the total gross moist static stability $\left(2.6 \times 10^{4} \mathrm{~J} \mathrm{~kg}^{-1}\right.$ compared to $2.0 \times 10^{4} \mathrm{~J} \mathrm{~kg}^{-1}$ in PD), weakens the canceling effect that the latent heat transport has on the DSE transport and yields a larger LGM total northward transport in the low latitudes compared to the present day. We speculate that this larger efficiency permits the LGM energy transport at low latitudes to respond more readily to the circulation changes imposed by the meridional temperature gradients. Further details behind the different Hadley cell responses in the two climates are beyond the scope of the study.

\subsection{Integrated surface budget response}

The atmospheric heat transport calculated above as the residual between the TOA and surface fluxes is the actual heat transported by the atmosphere since each atmospheric column must be in long-term energetic equilibrium. An implied ocean heat transport calculated analogously from the surface fluxes, on the other hand, represents the transport that would be necessary to uphold the SST field given the set of surface fluxes resulting from the experiment. One is thus implicitly assuming the SST field to be one of equilibrium, but given that the climate was perturbed away from the reference state and that the SSTs are fixed, there is little reason to expect this to actually be the case. To avoid confusion, it will thus be designated the integrated surface budget (ISB) in the following.

In our experiments, AHT responses occur because a highlatitude cooling, for instance, leads to a drop in the surfaceto-atmosphere energy flux. Unless the TOA flux responds in concert with the surface, this leads to a cooling of the atmospheric column, which continues until a new equilibrium has been attained between the decreased surface flux, increased atmospheric heat transport, and decreased TOA flux. As it turns out in the following, this atmospheric equilibrium tends to be one where the AHT responds much more strongly than the TOA flux, an effect which is manifested as Bjerknes compensation. The atmospheric heat transport response is thus a result of the combined surface flux and atmospheric changes per NHTD. The sensitivity of the surface fluxes to the imposed SST forcing may not be the same in the two climates and it is instructive to consider the integrated surface budget response per unit change in the NHTD.

At the equator (Fig. 10a), the ratio of the LGM and PD integrated surface budget slope equals 1.5 , while for the atmospheric heat transport (Fig. 2a), this ratio is 2.33, showing that in part the difference between the LGM and PD AHT response is due to the different atmosphere-surface flux interactions. This is even more pronounced at $30^{\circ} \mathrm{N}$ (Fig. 10b) where the integrated surface budget and atmospheric heat transport ratios of the LGM and PD slopes are the same (they equal 0.5) and it suggests that the doubled PD AHT response at $30^{\circ} \mathrm{N}$ is linked to the doubled PD ISB response. This is equivalent to saying that the atmosphere and surface flux changes yield the same degree of Bjerknes compensation in the two climates, a conclusion that can also be inferred by comparing the ISB to 
AHT sensitivities: the atmospheric heat transport compensates integrated surface budget changes by $0.22 / 0.28=0.79$ in LGM and $0.11 / 0.14=0.79$ in PD. As discussed above, both the surface flux and AHT changes result from a two-way atmosphere-surface interaction following the imposed SST changes. We find here that the magnitudes of these changes are such that there is a stronger stabilizing feedback by the combined response in PD than in LGM.

\section{Discussion and conclusion}

Our study demonstrates enhanced mid-latitude atmospheric heat transport sensitivity to the imposed high latitude surface temperature perturbations under warm (interglacial) compared to cold (glacial) conditions. This is shown to be a feature of both annual mean transports (in the experiments with added SST perturbations) and of monthly transports (in the control runs, due to seasonal variations of NHTD). The weakened glacial transport sensitivity is linked to a reduced transient eddy heat flux response. In comparison to the PD climate, the glacial climate is found to have stronger overall stationary eddy heat fluxes, weaker transient eddy heat fluxes, a stronger and narrower polar jet and decreased eddy kinetic energy in the North Atlantic. The transient eddy heat flux responses dominate over those of the stationary eddy heat fluxes, an effect which is larger in PD than in LGM simulations.

Consideration of the integrated surface flux budget reveals a significant role of the surface response in the upward propagation of the imposed SST forcing between the two climates, especially in the high latitudes where the TOA changes are small. Therefore, the effect found is a consequence of the combined surface-atmosphere interaction in which the final equilibrium surface and AHT response per NHTD is smaller in LGM than in PD. The atmosphere and surface fluxes react in a manner that provides a smaller negative feedback in the LGM per NHTD change.

Consequently, the PD climate appears more capable of damping high latitude anomalies due to the strong midlatitude response while much less change is seen in the tropics. In contrast, the LGM simulations display the major response in the tropics where the cancellation between dry static energy and latent heat transports is weaker and energy is transported more efficiently away from the Tropics. From Fig. 2 it is possible to estimate the sensitivity of transport convergence into the area $0-30^{\circ} \mathrm{N}$ for the PD and LGM experiments. When the NHTD increases by $1 \mathrm{~K}$ (as a result of the added surface perturbations), the PD equator sees an increased import of energy from the Southern Hemisphere of $0.09 \mathrm{PW}$ and at $30^{\circ} \mathrm{N}$ an increased poleward transport of $0.22 \mathrm{PW}$ is found. The area $0-30^{\circ} \mathrm{N}$ thus encounters a net loss of $0.13 \mathrm{PW}$. In the LGM experiments, the resulting import of energy from the Southern Hemisphere is considerably larger $(0.21 \mathrm{PW})$ and the poleward transport at $30^{\circ} \mathrm{N}$ is considerably smaller $(0.11 \mathrm{PW})$ and the area $0-30^{\circ} \mathrm{N}$ encounters a net gain of $0.10 \mathrm{PW}$. The two effects of the prime LGM response being in the tropics and the prime PD response being in the mid-latitudes thus combine to yield opposite tendencies on the $0-30^{\circ} \mathrm{N}$ energy budget with a gain in LGM and a loss in PD. The net contribution to the $30-90^{\circ} \mathrm{N}$ budgets from the energy transport are just equal to the $30^{\circ} \mathrm{N}$ sensitivities, i.e. $0.22 \mathrm{PW}$ for PD and $0.11 \mathrm{PW}$ for LGM. From these quantities, we can calculate the energy transport contribution to the tendency on NHTD as the net for the low latitude area minus that of the high latitude area. For the PD experiments we find $-0.13 \mathrm{PW}-0.22 \mathrm{PW}=-0.35 \mathrm{PW}$, and for the LGM we find $0.10 \mathrm{PW}-0.11 \mathrm{PW}=-0.01 \mathrm{PW}$. The contribution of the meridional energy transport to the negative tendency on the NHTD is thus much larger for the PD than for the LGM. In the PD, there is thus a much larger negative feedback on the imposed temperature gradients as a consequence of the enhanced atmospheric transport response.

Although the atmospheric circulation changes are not considered to be the primary cause of decreased interglacial variability, these results give an important indication of a varying ability of the surface and atmosphere to feed back negatively on imposed oceanic changes in different climates. Surface boundary conditions as seen by the atmosphere, i.e. topography, sea ice and SST, are quite different in PD and LGM, and the decreased LGM response we find is therefore a combination of all these factors. In order to qualitatively constrain the importance of the sea ice geometry, an additional experiment with three times the initial anomaly added to the LGM background climate was performed. This simulation had much less summer sea ice and a NHTD anomaly similar to that of the PD_p2 case. The overall slope of the LGM response was not affected significantly by the inclusion of this point, although if one considers only the warming LGM simulations (including the LM_p3 experiment), then the slopes in the two climates were more similar. Since these are all cases with less sea ice, the large sea ice extent in LGM simulations is possibly an important component in the decreased glacial energy transport feedback. This is, however, not necessarily the only reason, given that according to, for instance, Cook and Held (1988), it is the LGM orography that causes a change in the stationary eddies and thus forces a different partitioning of the transient and stationary components between the two climates.

In conclusion, the sensitivity of the atmospheric transport to NHTD changes, which depend on the background surface conditions described above, shows less negative transport feedback during the Last Glacial Maximum as compared to present day. Choosing the prescribed SST configuration in our experiments enabled the study of the atmosphere only response to the applied perturbation, but did not allow for ocean feedbacks. Further work is required to evaluate the complete long term response when interactions with the ocean surface are allowed. Presumably, such interactions could tend to reduce the effect of the meridional transport feedback discussed here. 
Acknowledgements. This work was supported in part by a grant of HPC resources from the Arctic Region Supercomputing Center (ARSC) at the University of Alaska Fairbanks as part of the Department of Defense High Performance Computing Modernization Program. The authors acknowledge the support of the Danish National Research Foundation and thank Peter D. Ditlevsen and John C. H. Chiang for useful discussions as well as Peter Wang for supplying the temperature anomalies associated with the ECBilt-CLIO freshwater experiments.

Edited by: U. Mikolajewicz

\section{References}

Banakar, V. K., Mahesh, B. S., Burr, G., and Chodankar, A. R.: Climatology of the Eastern Arabian Sea during the last glacial cycle reconstructed from paired measurement of foraminiferal $\delta^{18} \mathrm{O}$ and $\mathrm{Mg} / \mathrm{Ca}$, Quaternary Res., 73/3, 535-540, doi:10.1016/j.yqres.2010.02.002, 2010.

Broccoli, A. J., Dahl, K. A., and Stouffer, R. J.: Response of the ITCZ to Northern Hemisphere cooling, Geophys. Res. Lett., 33, L01702, doi:10.1029/2005GL024546, 2006.

Broecker, W. S.: Massive iceberg discharges as triggers for global climate change, Nature, 372, 421-424, 1994.

Caballero, R. and Langen, P. L.: The dynamic range of poleward energy transport in an atmospheric general circulation model, Geophys. Res. Lett., 32, L02705, doi:10.1029/2004GL021581, 2005.

Cheng, W., Bitz, C. M., and Chiang, J. C. H.: Adjustment of the global climate to an abrupt slowdown of the Atlantic meridional overturning circulation, in: Ocean Circulation: Mechanisms and Impacts, Geophys. Monogr. Ser., vol. 173, edited by: Schmittner, A., Chiang, J. C. H., and Hemming, S. R., AGU, Washington, DC, 295-313, 2007.

Chiang, J. C. H. and Bitz, C. M.: Influence of high latitude ice cover on the marine Intertropical Convergence Zone, Clim. Dynam., 25, 477-496, 2005.

Chiang, J. C. H., Cheng, W., and Bitz, C. M.: Fast teleconnections to the tropical Atlantic sector from Atlantic thermohaline adjustment, Geophys. Res. Lett., 35, L07704, doi:10.1029/2008GL033292, 2008.

CLIMAP: CLIMAP 18K Database, IGBP PAGES/World Data Center for Paleoclimatology Data Contribution Series 94-001, Technical report NOAA/NGDC Paleoclimatology Program, Boulder CO, USA, 1994.

Cook, K. H. and Held, I. M.: Stationary waves of the ice age climate, J. Climate, 1, 807-819, 1988.

Dahl, K. A., Broccoli, A. J., and Stouffer, R. J.: Assessing the role of North Atlantic freshwater forcing in millennial scale climate variability: A tropical Atlantic perspective, Clim. Dynanm., 24, 325-346, 2005.

Donohoe, A. and Battisti, D. S.: Causes of reduced North Atlantic storm activity in a CAM3 simulation of the Last Glacial Maximum, J. Climate, 22, 4793-4808, 2009.

EPICA Community Members: One-to-one coupling of glacial climate variability in Greenland and Antarctica, Nature, 444, 195198, 2006.
Goosse, H. and Fichefet, T.: Importance of ice-ocean interactions for the global ocean circulation: A model study, J. Geophys. Res., 104, 1999, 23337-23355, 1999.

Hurrell, J. W., Hack, J. J., Boville, B. A., Williamson, D. L., and Kiehl, J. T.: The Dynamical Simulation of the NCAR Community Climate Model Version 3 (CCM3)*, J. Climate, 11, 12071236, doi:1520-0442(1998)011;1207:TDSOTN $i 2.0 . C O ; 2$, 1998.

Hwang, Y. T. and Frierson, D. M. W.: Increasing atmospheric poleward energy transport with global warming, Geophys. Res. Lett., 37, L24807, doi:10.1029/2010GL045440, 2010.

Kang, S. M., Frierson, D. M. W., and Held, I. M.: The tropical response to extratropical thermal forcing in an idealized GCM: The importance of radiative feedbacks and convective parameterization, J. Atmos. Sci., 66, 2812-2827, doi:10.1175/2009JAS2924.1, 2009.

Keigwin, L. D., Curry, W. B., Lehman, S. J., and Johnsen, S.: The role of the deep ocean in North Atlantic climate change between 70 and $130 \mathrm{kyr}$ ago, Nature, 371, 323-326, 1994.

Kiehl, J. T., Hack, J. J., Bonan, G. B., Boville, B. A., Williamson, D. L., and Rasch, P. J.: The National Center for Atmospheric Research Community Climate Model: CCM3, J. Climate, 11, 1131-1149, 1998.

Li, C. and Battisti, D. S.: Reduced Atlantic Storminess during Last Glacial Maximum: Evidence from a Coupled Climate Model, J. Climate, 21, 3561-3579, 2008.

Magnusdottir, G. and Saravanan, R. : The response of atmospheric heat transport to zonally averaged SST trends, Tellus A, 51, 815832, doi:10.1034/j.1600-0870.1999.00019.x, 1999.

Murakami, S., Ohgaito, R., Abe-Ouchi, A., Crucifix, M., and OttoBliesner, B. L.: Global-Scale Energy and Freshwater Balance in Glacial Climate: A Comparison of Three PMIP2 LGM Simulations, J. Climate, 21, 5008-5033, 2008.

NGRIP members: High-resolution record of Northern Hemisphere climate extending into the last interglacial period, Nature, 432, 147-151, 2004.

Opsteegh, J. D., Haarsma, R. J., Selten, F. M., and Kattenberg, A.: ECBILT: a dynamic alternative to mixed boundary conditions in ocean models, Tellus A, 50, 348-367, 1998.

Peixoto, J. P. and Oort, A. H.: "Physics of climate", New York, USA: American Institute of Physics, ISBN 088318712 4, 520 pp., 1992.

Peltier, W. R.: Global glacial isostasy and the surface of the ice age Earth: The ICE-5G (VM2) model and GRACE, Ann. Rev. Earth Planet. Sci., 32, 111-149, 2004.

Peterson, L. C., Haug, G. H., Hughen, K. A., and Rohl, U.: Rapid changes in the hydrologic cycle of the tropical Atlantic during the last glacial, Science, 290, 1947-1951, doi:10.1126/science.290.5498.1947, 2000.

Renssen, H., Goose, H., and Fichefet, T.: Modeling the effect of freshwater pulses on the early Holocene climate: The influence of high-frequency climate variability, Paleoceanography, 17, 1020, doi:10.1029/2001PA000649, 2002.

Shea, D. J., Trenberth, K. E., and Reynolds, R. W.: A global monthly sea surface temperature climatology, J. Climate, 5, 9871001, 1992.

Trenberth, K. E. and Caron, J. M.: Estimates of meridional atmosphere and ocean heat transports, J. Climate, 14, 3433-3443, 2001. 
Trenberth, K. E. and Stepaniak, D. P.: Covariability of components of poleward atmospheric energy transports on seasonal and interannual timescales, J. Climate, 16, 3691-3705, 2003.

Trenberth, K. E., Caron, J. M., and Stepaniak, D. P.: The atmospheric energy budget and implications for surface fluxes and ocean heat transports, Clim. Dynam, 17, 259-276, 2000.

Wang, P.: Seasonality over Greenland during the Holocene and possible explanations of the $8.2 \mathrm{kyr}$ event - A study based on the circulation model ECBilt-CLIO, Msc Thesis, NBI, University of Copenhagen, Denmark, 2009.
Wang, Y. J., Cheng, H., Edwards, R. L., An, Z. S., Wu, J. Y., Shen, C. C., and Dorale, J. A.: A High-Resolution Absolute-Dated Late Pleistocene Monsoon Record from Hulu Cave, China, Science, 294, 2345-2348, doi:10.1126/science.1064618, 2001.

Wunsch, C.: The total meridional heat flux and its oceanic and atmospheric partition, J. Climate, 18, 4374-4380, 2005.

Zhang, R. and Delworth, T. L.: Simulated tropical response to a substantial weakening of the Atlantic thermohaline circulation, J. Climate, 18, 1853-1860, 2005. 